\title{
$1 \quad$ Validating marker-less pose estimation with 3D x-ray radiography
}

2 Dalton D. Moore ${ }^{1}$, Jeffrey D. Walker ${ }^{2}$, Jason N. MacLean ${ }^{1,4,5}$, Nicholas G. Hatsopoulos ${ }^{1,2,5}$

$4 \quad{ }^{1}$ Committee on Computational Neuroscience, University of Chicago, Chicago, Illinois;

$5 \quad{ }^{2}$ Department of Organismal Biology and Anatomy, University of Chicago, Chicago, Illinois;

$6{ }^{4}$ Department of Neurobiology, University of Chicago, Chicago, Illinois;

$7 \quad{ }^{5}$ University of Chicago Neuroscience Institute, Chicago, Illinois

9 Running Title: Validating marker-less pose estimation

10 Corresponding Author: nicho@uchicago.edu

11 Keywords: DeepLabCut, marker-less tracking, marmoset, kinematics, XROMM, pose

12 estimation

13

14 Summary Statement: We validate the accuracy of DeepLabCut, a widely used marker-less

15 pose estimation tool, using a marker-based 3D x-ray radiography system (XROMM). 


\section{ABSTRACT}

17 To reveal the neurophysiological underpinnings of natural movement, neural recordings must be

18 paired with accurate tracking of limbs and postures. Here we validate the accuracy of

19 DeepLabCut (DLC) by comparing it to a 3D x-ray video radiography system that tracks markers

20 placed under the skin (XROMM). We record behavioral data simultaneously with XROMM and

21 high-speed video for DLC as marmosets engage in naturalistic foraging and reconstruct three-

22 dimensional kinematics in a shared coordinate system. We find that DLC tracks position and

23 velocity of 12 markers on the forelimb and torso with low median error $(0.272 \mathrm{~cm}$ and $1.76 \mathrm{~cm} / \mathrm{s}$,

24 respectively) corresponding to $2.9 \%$ of the full range of marker positions and $5.9 \%$ of the range

25 of speeds. For studies that can accept this relatively small degree of error, DLC and similar

26 marker-less pose estimation tools enable the study of more naturalistic, unconstrained

27 behaviors in many fields including non-human primate motor control. 


\section{INTRODUCTION}

As the study of motor neuroscience progresses toward an emphasis on naturalistic, unconstrained behavior, kinematics must be captured accurately and efficiently. Past research has relied on marker-based systems that track markers attached to an animal's skin (such as Vicon, OptiTrack, and PhaseSpace) or surgically implanted radiopaque beads (XROMM; Brainerd et al. 2010). However, these systems are expensive and can be impractical with smaller species like mice or marmosets; they become increasingly impractical if the study focuses on freely-moving behaviors. To solve this problem, multiple groups have developed marker-less pose estimation tools that use deep learning to apply digital markers to recorded video. The most widely used is DeepLabCut (DLC; Mathis et al. 2018), but multiple alternatives exist (Graving et al., 2019; Pereira et al., 2019; Wu et al., 2020). These enable the study of a wider range of behaviors by allowing the subject to move without the disturbance of physical markers. Furthermore, the use of artificial neural networks removes the bottleneck of tracking physical markers semi-automatically; a well-trained, robust network can label video effectively with minimal hands-on time. DLC has been used in a variety of contexts, including tracking eye movements and pupil dilation (Siegle et al., 2019; Steinmetz et al., 2019), hand movements (Sauerbrei et al., 2020), and gross position (Comer et al., 2020) of mice, all with accuracy comparable to human labelers. However, to our knowledge DLC accuracy has not yet been compared to that of marker-based tracking (but see Pouw, Trujillo, and Dixon 2020 for validation in gesture-speech synchrony as compared to marker-based tracking). Such a comparison is crucial to determine whether DLC can reliably track kinematics with accuracy similar to existing marker-based systems and to understand the sources of error that can be ameliorated in future work. XROMM provides a useful basis for comparison, as we have shown that the system tracks radiopaque markers with $0.06 \mathrm{~mm}$ precision based on the standard deviation of intermarker distances during a recording of a calibration specimen (Walker et al., 2020). To this end, we collect simultaneous recordings with XROMM and video (two FLIR Blackfly S cameras at 200 frames per second) as common marmosets engage in naturalistic foraging, then reconstruct three-dimensional reaching kinematics in a shared coordinate system. We find that DLC tracks position and velocity with a median absolute error of $0.272 \mathrm{~cm}$ (mean absolute error of $0.344 \mathrm{~cm})$ and $1.76 \mathrm{~cm} / \mathrm{s}(2.50 \mathrm{~cm} / \mathrm{s})$, respectively. These errors are $2.9 \%$ of the full range of marker positions and $5.9 \%$ of the range in speeds. We then discuss sources of error and ways by which this error can be reduced or eliminated. 


\section{METHODS}

\section{Subjects}

63 These experiments were conducted with two common marmosets (Callithrix jacchus) (an 8-year

64 old, $356 \mathrm{~g}$ male and a 7-year old, $418 \mathrm{~g}$ female). All methods were approved by the Institutional

65 Animal Care and Use Committee of the University of Chicago.

\section{Data Collection}

67 The two marmosets were placed together in a $1 \mathrm{~m} \times 1 \mathrm{~m} \times 1 \mathrm{~m}$ cage with a modular foraging 68 apparatus attached to the top of the cage, as previously described by Walker et al. (2020). The 69 marmosets were allowed to forage voluntarily throughout recording sessions that lasted 1-2 70 hours. Individual trials were triggered manually with a foot pedal by the experimenters when the 71 marmosets appeared ready to initiate a reach. The manual trigger initiated synchronized video 72 collection by the XROMM system (Brainerd et al., 2010) and two visible light cameras, each 73 described in further detail below. We retained all trials that captured right-handed reaches.

\section{XROMM}

75 Bi-planar X-ray sources and image intensifiers $(90 \mathrm{kV}, 25 \mathrm{~mA}$ at $200 \mathrm{fps})$ were used to track the $763 \mathrm{D}$ position of radiopaque tantalum beads $(0.5-1 \mathrm{~mm}$, Bal-tec) placed subcutaneously in the 77 arm, hand, and torso. Details of bead implants can be found in Walker et al. (2020), in which the 78 authors also report estimating XROMM marker tracking precision of $0.06 \mathrm{~mm}$. Positions of 13 79 beads were tracked using a semi-automated process in XMALab (Knorlein et al., 2016). One 80 bead implanted in the torso was ignored for analysis later to match the 12 labels in DLC.

\section{DeepLabCut}

82 Two high-speed cameras (FLIR Blackfly S, 200 fps, 1440x1080 resolution) were used to record

83 video for analysis by DLC. The cameras were positioned to optimize visibility of the right upper

84 limb during reaching behavior in the foraging apparatus and to minimize occlusions, while

85 avoiding the path between the X-ray sources and image intensifiers (Fig. 1A). The cameras

86 were triggered to record continuous images between the onset and offset of the manual

87 XROMM trigger, with series of images later converted to video for DLC processing. We labeled

8812 body parts in DLC - three labels on each of the torso, upper arm, forearm, and hand (Fig.

89 1B). We used DLC 2.0.7 with in-house modifications to produce epipolar lines in image frames

90 that were matched between the two cameras (Fig. 1C), which significantly improved human

91 labeling accuracy. This modification has since been added as a command line feature in the 
92 DLC package. Aside from this and related changes to the standard DLC process, we followed

93 the steps outlined in Nath et al. (2019).

\section{Calibration}

95 A custom calibration device was built to allow for calibration in both recording domains (Knorlein et al. 2016; instruction manual for small lego cube is located in the XMALab BitBucket). The device was constructed from LEGO bricks and Sugru putty, with a three-dimensional grid of steel beads within the structure and a two-dimensional grid of white circles on one face of the cube. Calibration of $x$-ray images was computed in XMALab and calibration of visible light images was computed with custom code using OpenCV. This integrated calibration device ensures that DLC and XROMM produced tracked trajectories in a common 3D coordinate

102 system.

\section{Training network and experimental design}

104 We trained two separate networks to label marmoset body parts - one for each marmoset.

105 Marmoset TY produced four useful reaching events containing 1-3 reaches each and marmoset 106 PT produced 13 reaching events. We extracted 100 total frames (50/camera) across the four 107 events for marmoset TY and 254 frames (127/camera) across seven events for marmoset PT, 108 although we later removed one these (and its eight pairs of images) and one of the unlabeled 109 events from the data because they each contained a single reach with so many occlusions that 110 they could not be adequately tracked without extensive refinement. After preprocessing 111 (discussed below), our dataset consisted of 5721 frames in the test set and 169 frames in the 112 train set. All 169 training frames came from four TY events and six PT events, while the test 113 frames came from those same events as well as five PT events that were completely held-out 114 from the training set.

\section{Label refinement}

116 Both networks labeled reaching events well in the case that the reach trajectory was 117 represented in the training data. As documented by the DLC authors, the networks failed in 118 sections of reaches that were similar to poorly labeled training images or sections of reaches 119 completely unrepresented in the training set. After the first iteration of video analysis was 120 completed and evaluated, we used the DLC refinement tools to extract outlier frames in poorly 121 labeled videos and improve the training set before retraining the network. We went through the 122 refinement process one time. 


\section{Trajectory processing}

124 Because we focused on labeling periods of active reaching, large portions of video that capture

125 the marmoset chewing or otherwise disengaged from the foraging task were poorly labeled by

126 the DLC networks. In order to isolate reaching movements, we first removed all frames for

127 which the label's DLC likelihood score was below 0.05 . We then removed all frames for which

128 the lateral hand label was well behind the foraging partition and retained the rest of the frames.

129 The remaining frames correspond to reaching movements within the foraging arena and brief

130 periods of rest preceding, between, and after reaching movements. DLC trajectories were

131 smoothed using a $3^{\text {rd }}$ order Savitsky-Golay filter with a 31-sample sliding window. Finally, we

132 found that there was a brief delay ranging from 0 to 10 frames between pedal-triggered onset of

133 the XROMM event and the corresponding pedal-triggered TTL pulse initiating the start of the

134 event for the FLIR cameras (and for the pulse ending the event). To adjust for the timing

135 difference, we iterated over a range of possible sample shifts separately for each event to find

136 the shift that minimized the mean absolute error between DLC and XROMM trajectories. We

137 visually inspected each trajectory after the adjustment to ensure the shift was qualitatively

138 accurate.

\section{Evaluation of DLC Accuracy}

140 We computed the median and mean absolute error between the position and velocity traces

141 found using DLC and the matched XROMM trajectories for all body parts across all reaching

142 events. Body parts were ignored for instances in which they were obscured from view by the

143 visible light cameras for an extended period as a result of the second marmoset entering the

144 camera view mid-reach. This applied to the torso markers in one event and all but the hand

145 markers in another event.

\section{Statistical Tests}

147 Since the error distributions are right-skewed with long tails of large errors, we use the median

148 error to describe the center of each distribution and the Mann-Whitney U-Test to assess

149 statistical significance. The P-values computed with this method are artificially low due to the

150 large sample size (e.g. 17,671 samples for the three hand markers and 14,986 samples for the

151 three torso markers), so we report the correlation effect size defined by the rank-biserial

152 correlation to describe statistical differences between distributions. According to convention, we

153 consider $r<0.20$ to be a negligible effect (Cohen, 1992). 


\section{Normalized Error and Fraction of Variance Accounted For}

155 To compute normalized error, we divided the position and velocity errors by the maximum range 156 of motion and maximum speed for each marker across the dataset. To compute the fraction of 157 variance accounted for, we used the following equation:

$$
1-\frac{\sum(D L C-X R O M M)^{2}}{\sum[X R O M M-\operatorname{mean}(X R O M M)]^{2}}
$$

which normalizes the sum of squared DLC error by the XROMM variance and subtracts from one.

\section{RESULTS}

\section{Position and Velocity Error - Tracking Examples}

164 Qualitatively, DLC and XROMM-tracked forelimb trajectories were nearly identical for most time 165 points and reaches (Fig. 2A-C). The 3D view shows DLC tracking the overall motion and most 166 fine details accurately, while missing some brief position changes tracked by XROMM near the 167 start of the reach (Fig. 2A). Breaking out $x-y-z$ components demonstrates minimal position and 168 velocity error (Fig. 2B,C), with median error of $0.238 \mathrm{~cm}$ and $2.25 \mathrm{~cm} / \mathrm{s}$ (mean error of 0.237 and $1692.70 \mathrm{~cm} / \mathrm{s}$ ) for this event.

170 Some reaches were not tracked with the same accuracy, with large errors falling into a few 171 categories. One category is exemplified in Fig. 2D-F, in which a marker jump that occurred 172 around 1.25s was removed during DLC processing and replaced with a linear interpolation (Fig. $1732 \mathrm{E}, \mathrm{F})$. This results in a spike of position and velocity error and correspondingly high average 174 errors (median of $0.464 \mathrm{~cm}$ and mean of $0.488 \mathrm{~cm}$ for position, median of $1.37 \mathrm{~cm} / \mathrm{s}$ and mean 175 of $2.88 \mathrm{~cm} / \mathrm{s}$ for velocity). A second category of errors occurred when markers moved rapidly 176 away from the correct location over a few frames at the beginning or end of tracking. The 177 majority of these large errors occurred because the training dataset did not contain frames that 178 were similar enough to the problematic reaching periods (see Discussion). Finally, some of the 179 less extreme errors above the median occurred due to human labeling inconsistency, 180 particularly when visual landmarks were unclear or changed due to skin deformation.

\section{Position and Velocity Error - Aggregate Results}


We found that DLC tracked position and velocity with median error of $0.272 \mathrm{~cm}$ (mean of 0.344 $\mathrm{cm})$ and $1.76 \mathrm{~cm} / \mathrm{s}(2.49 \mathrm{~cm} / \mathrm{s})$, respectively (Fig. 3A,B, right). Position and velocity error distributions were right-skewed with a long tail of large errors, so we focused on median error as the measure of accuracy. We found that position errors for the forearm (median of $0.381 \mathrm{~cm}$ ) and torso $(0.328 \mathrm{~cm})$ were significantly larger than errors for hand $(0.217 \mathrm{~cm})$ and upper arm $(0.215 \mathrm{~cm})$ markers (Mann Whitney U-test; $r_{\text {forearm-hand }}=0.42, r_{\text {forearm-upperArm }}=0.46, r_{\text {torso-hand }}=$ $0.34, r_{\text {torso-upperArm }}=0.38$ ). Velocity errors varied between body segments to a lesser degree - the only modest difference was higher error for the torso than the upper arm (medians of $1.93 \mathrm{~cm} / \mathrm{s}$ and $1.47 \mathrm{~cm} / \mathrm{s}, r=0.23$ ). We note that the forearm and torso were more difficult to label manually than the hand and upper arm. As such, we attribute variation in tracking quality across body segments to human labeling inconsistency rather than any issues with DLC itself; we suggest taking the hand and upper arm errors as more indicative of DLC performance.

To provide context, we normalized position and velocity errors by the maximum range of position and speed for each marker. We found that the median error across events and markers was just $2.9 \%$ of position range and $5.9 \%$ of the speed range. The hand markers, for which visual landmarks were most clear, had median errors equivalent to $1.9 \%$ and $3.2 \%$ of position and speed ranges. Viewed another way, we find that DLC accounts for $94.1 \%$ of the XROMM position variance and $81.2 \%$ of velocity variance for all data points, or $96.5 \%$ and $89.6 \%$, respectively, when we removed errors above the 0.975 quantile to focus on well-labeled data.

We separated the 169 datapoints that correspond to image frames labeled by hand (train) from the rest of the data which were labeled by the network only (test) and report the median error across three markers on each body segment (Fig. 3A,B, left). Median position error was $0.32 \mathrm{~cm}$ for train and $0.27 \mathrm{~cm}$ for test, but this effect was negligible $(r=0.11)$. Velocity train errors were significantly greater $($ median $=2.40 \mathrm{~cm} / \mathrm{s})$ than test $(1.75 \mathrm{~cm} / \mathrm{s})$ with $r=0.22$. The hand $(2.48 \mathrm{~cm} / \mathrm{s}$ to $1.61 \mathrm{~cm} / \mathrm{s}, \mathrm{r}=0.27)$ and forearm $(3.21 \mathrm{~cm} / \mathrm{s}$ to $2.17 \mathrm{~cm} / \mathrm{s}, \mathrm{r}=0.29)$. drove this result, while the effect was negligible for the upper arm and torso.

\section{Inter-Marker Distances}

209 We estimated the precision of tracking by the standard deviation of inter-marker distances within 210 each body segment, with the expectation that inter-marker distances should be near constant 211 aside from soft tissue deformation. We report precision of $0.21 \mathrm{~cm}$ for the hand and forearm, $2120.22 \mathrm{~cm}$ for the upper arm, and $0.37 \mathrm{~cm}$ for the torso. This is of similar magnitude to the position 213 error and results from variability in human labeling and soft tissue deformation. 


\section{DISCUSSION}

215 We have shown that DLC tracks movements of marmoset forelimb and torso accurately in 216 comparison to marker-based tracking with XROMM. The median errors of $0.272 \mathrm{~cm}$ and 1.76

$217 \mathrm{~cm} / \mathrm{s}(2.9 \%$ and $5.9 \%$ of kinematic range) are sufficient for many purposes, and there is reason

218 to treat this as an upper error bound for well-trained DLC networks. These errors should provide 219 an expectation of the accuracy that can be attained with a simple recording setup and relatively

220 little computer vision experience, but which could be improved with targeted adjustments.

\section{Assessment of Errors and Limitations}

222 We present these results as an upper bound on DLC error due to some limitations in the 223 dataset. The first is the small number of events due to difficulties associated with the XROMM; 224 the marmosets were uncomfortable in the space and therefore less interested in foraging and it 225 was difficult to manually trigger data collection at the onset of voluntary movements. The second 226 limitation is the steep angle recorded with DLC camera-2 (Fig. 1), which would ideally have 227 been placed in the center of the image intensifier rather than mounted on top - this made 228 identifying visual landmarks difficult, particularly on the torso where only a fraction of potential 229 landmarks was visible in most frames. Finally, the useful landmarks on the forearm did not align 230 well with estimated XROMM bead positions on the DLC images and were difficult to locate 231 when the marmoset made short reaches to the left corner of the foraging tray. Labeling of the 232 hand and upper arm was not as affected by the camera angles and ambiguous landmarks, 233 which explains the lower error for these body segments.

234 DLC performance is dependent on the quality and breadth of training data - movements that 235 are sparsely or un-represented in training data are unlikely to be well-tracked by the network. 236 Tracking errors such as in Fig. 2D-F can be reduced by labeling a comprehensive dataset from 237 the outset or by rigorous relabeling of problematic video frames using the DLC toolbox.

\section{Improving Accuracy}

239 DLC tracking can be made more accurate by improving data quality, improving human labeling 240 performance, and utilizing processing tools within and outside of DLC. Image quality is the 241 fundamental limiting factor to DLC accuracy - resolution affects the number of pixels per unit 242 distance, while resolution, visibility, contrast, and brightness constrain human labeling 243 consistency. We suggest recording at the highest feasible resolution and using more than two 244 cameras to improve coverage and reduce occlusions, as well as increase the benefit of 3D 
245 processing tools discussed below. Additionally, lighting equipment and consideration of

246 occlusions and visible landmarks on the animal can improve human labeling.

247 After collecting high-quality data, there are tools available to improve tracking accuracy. Most 248 proximally, DLC is open-source and actively maintained, with frequent implementation of new

249 features. We have contributed one such feature by incorporating epipolar lines to improve 250 labeling precision across cameras for 3D projects (Fig. 1C). Epipolar lines simplify the 251 identification of the same landmark in different camera angles and are especially helpful when

252 landmarks are ambiguous. At time of publication, this has been implemented for pairs of 253 cameras but could be extended such that epipolar lines from two cameras provide a 254 triangulated point in subsequent views.

255 Additional tools operate as add-ons to base DLC, including anipose (Karashchuk et al., 256 2020), pose3d (Sheshadri et al., 2020), OpenMonkeyStudio (Bala et al., 2020), and a new 257 development that uses 3D modeling to create synthetic training data (Bolaños et al., 2021).

258 Anipose and pose3d facilitate 3D pose estimation with more than 2 cameras and standardized 259 filtering options. Anipose provides an especially useful 3D-filtering option that improves 260 performance over the standard process of filtering in 2D before triangulation to 3D coordinates. 261 OpenMonkeyStudio and the Bolaños et al. (2021) method are data augmentation tools - the 262 former uses labels on a subset of 62 camera views to produce triangulated labels on remaining 263 views, while the latter animates a 3D model to render photo-realistic images and labels as 264 synthetic training data for DLC.

\section{Applicability to other animal pose estimation tools}

266 Although DLC is the most widely used marker-less pose estimation tool, there are a few 267 alternatives. LEAP (Pereira et al., 2019) and DeepPoseKit (Graving et al., 2019) use different 268 network architectures to reduce training and inference time. Deep Graph Pose (DPG; Wu et al. 269 2020) uses the same architecture as DLC but implements temporal and spatial constraints to 270 reduce the necessary training set size and eliminate marker jumps. However, the authors do not 271 compare performance for the frames without marker jumps, which don't seem to differ from DLC 272 in example trials they present. This suggests that DPG reduces the long tail of high-error 273 frames, but it is unclear whether it improves accuracy for well-tracked frames. Thus, we expect 274 DLC errors reported here to be representative for existing tools, at least for the subset of well275 tracked frames. 


\section{Future Work}

278 We plan to extend DLC outputs to reconstruct joint angular motion about the shoulder, elbow, 279 and wrist in a joint coordinate system following Walker et al. (2020), by adapting the workflow 280 described by Brainerd et al. (2010). However, dealing with inter-marker distance jitter and estimating the position of DLC labels on 3D-rendered CT-scan models has proven challenging. We hope to address these problems and decrease "human" labeling inconsistency by generating synthetic training data with a 3D marmoset model (similar to the mouse model described by Bolaños et al. 2021) and applying DLC outputs with this marmoset model to constrain the inverse kinematics computations of joint angular motion.

\section{ACKNOWLEDGEMENTS}

288 We thank Marina Sundiang for early help setting up data collection and for contributions to animal care and training; J.D. Laurence-Chasen for help building the Lego calibration object; the XROMM Technology Development Project for the XROMM; Ben Knorlein for XMALab and assistance locating pertinent code for rigid body transformations in the XMALab code; and Alexander Mathis and Mackenzie Mathis for DeepLabCut. This is University of Chicago XROMM Facility Publication 8.

\section{COMPETING INTERESTS}

295 The authors declare no competing or financial interests.

\section{AUTHOR CONTRIBUTIONS}

297 D.D.M, J.D.W., J.N.M. and N.G.H. developed the initial concept of validating DLC with XROMM.

298 D.D.M. and J.D.W. set up and optimized cameras for DLC and collected all data. D.D.M.

299 analyzed all the data and wrote all the code, with significant help and input from J.D.W.; D.D.M.

300 wrote the manuscript and all authors read the manuscript, provided critical comments for

301 revision and approved the final version. J.N.M. and N.G.H. provided guidance at each phase of

302 the process. D.D.M., J.D.W., J.N.M. and N.G.H. acquired funding.

\section{FUNDING}

304 This work was supported by the National Institutes of Health through a Brain Initiative Grant 305 (R01NS104898) and through an NRSA F31 fellowship (1F31NS118950-01; D.D.M.). Funding 
bioRxiv preprint doi: https://doi.org/10.1101/2021.06.15.448541; this version posted June 16, 2021. The copyright holder for this preprint (which was not certified by peer review) is the author/funder. All rights reserved. No reuse allowed without permission.

306 for the UChicago XROMM Facility was provided by National Science Foundation Major

307 Research Instrumentation Grants MRI 1338036 and 1626552.

\section{DATA AVAILABILITY}

309 Data available upon request. Code available at https://github.com/hatsopoulos-lab/marmoset-

310 dlc_xromm_validation. 


\section{REFERENCES}

Bala, P. C., Eisenreich, B. R., Yoo, S. B. M., Hayden, B. Y., Park, H. S. and Zimmermann, J. (2020). Automated markerless pose estimation in freely moving macaques with OpenMonkeyStudio. Nat. Commun. 11, 1-12.

Bolaños, L. A., Xiao, D., Ford, N. L., LeDue, J. M., Gupta, P. K., Doebeli, C., Hu, H., Rhodin, H. and Murphy, T. H. (2021). A three-dimensional virtual mouse generates synthetic training data for behavioral analysis. Nat. Methods 18, 378-381.

Brainerd, E. L., Baier, D. B., Gatesy, S. M., Hedrick, T. L., Metzger, K. A., Gilbert, S. L. and Crisco, J. J. (2010). X-ray reconstruction of moving morphology (XROMM): precision, accuracy and applications in comparative biomechanics research. J. Exp. Zool. Part A Ecol. Genet. Physiol. 313, 262-279.

Cohen, J. (1992). A power primer. Psychol. Bull. 112, 155-159.

Comer, A. L., Jinadasa, T., Sriram, B., Phadke, R. A., Kretsge, L. N., Nguyen, T. P. H., Antognetti, G., Gilbert, J. P., Lee, J., Newmark, E. R., et al. (2020). Increased expression of schizophrenia-associated gene C4 leads to hypoconnectivity of prefrontal cortex and reduced social interaction. PLOS Biol. 18, e3000604.

Graving, J. M., Chae, D., Naik, H., Li, L., Koger, B., Costelloe, B. R. and Couzin, I. D. (2019). DeepPoseKit, a software toolkit for fast and robust animal pose estimation using deep learning. Elife 8, e47994.

Karashchuk, P., Rupp, K. L., Dickinson, E. S., Sanders, E., Azim, E., Brunton, B. W. and Tuthill, J. C. (2020). Anipose: A toolkit for robust markerless 3D pose estimation. bioRxiv 117325.

Knorlein, B. J., Baier, D. B., Gatesy, S. M., Laurence-Chasen, J. D. and Brainerd, E. L. (2016). Validation of XMALab software for Marker-based XROMM. J. Exp. Biol. 219, 37013711.

Mathis, A., Mamidanna, P., Cury, K. M., Abe, T., Murthy, V. N., Mathis, M. W. and Bethge, M. (2018). DeepLabCut: markerless pose estimation of user-defined body parts with deep learning. Nat. Neurosci. 21, 1281-1289.

Nath, T., Mathis, A., Chen, A. C., Patel, A., Bethge, M. and Mathis, M. W. (2019). Using DeepLabCut for 3D markerless pose estimation across species and behaviors. Nat. Protoc. 
14, 2152-2176.

Pereira, T. D., Aldarondo, D. E., Willmore, L., Kislin, M., Wang, S. S.-H., Murthy, M. and Shaevitz, J. W. (2019). Fast animal pose estimation using deep neural networks. Nat. Methods 16, 117-125.

Pouw, W., Trujillo, J. P. and Dixon, J. A. (2020). The quantification of gesture-speech synchrony: A tutorial and validation of multimodal data acquisition using device-based and video-based motion tracking. Behav. Res. Methods 52, 723-740.

Sauerbrei, B. A., Guo, J. Z., Cohen, J. D., Mischiati, M., Guo, W., Kabra, M., Verma, N., Mensh, B., Branson, K. and Hantman, A. W. (2020). Cortical pattern generation during dexterous movement is input-driven. Nature 577, 386-391.

Sheshadri, S., Dann, B., Hueser, T. and Scherberger, H. (2020). 3D reconstruction toolbox for behavior tracked with multiple cameras. J. Open Source Softw. 5, 1849.

Siegle, J. H., Jia, X., Durand, S., Gale, S., Bennett, C., Graddis, N., Heller, G., Ramirez, T. K., Choi, H., Luviano, J. A., et al. (2019). A survey of spiking activity reveals a functional hierarchy of mouse corticothalamic visual areas. bioRxiv 805010.

Steinmetz, N. A., Zatka-Haas, P., Carandini, M. and Harris, K. D. (2019). Distributed coding of choice, action and engagement across the mouse brain. Nature 576, 266-273.

Walker, J. D., Pirschel, F., Gidmark, N., MacLean, J. N. and Hatsopoulos, N. G. (2020). A platform for semiautomated voluntary training of common marmosets for behavioral neuroscience. J. Neurophysiol. 123, 1420-1426.

Wu, A., Buchanan, E. K., Whiteway, M., Schartner, M., Meijer, G., Norovich, A., Noel, J. P., Schaffer, E., Rodriguez, E., Mishra, N., et al. (2020). Deep Graph Pose: A semisupervised deep graphical model for improved animal pose tracking. bioRxiv 259705. 

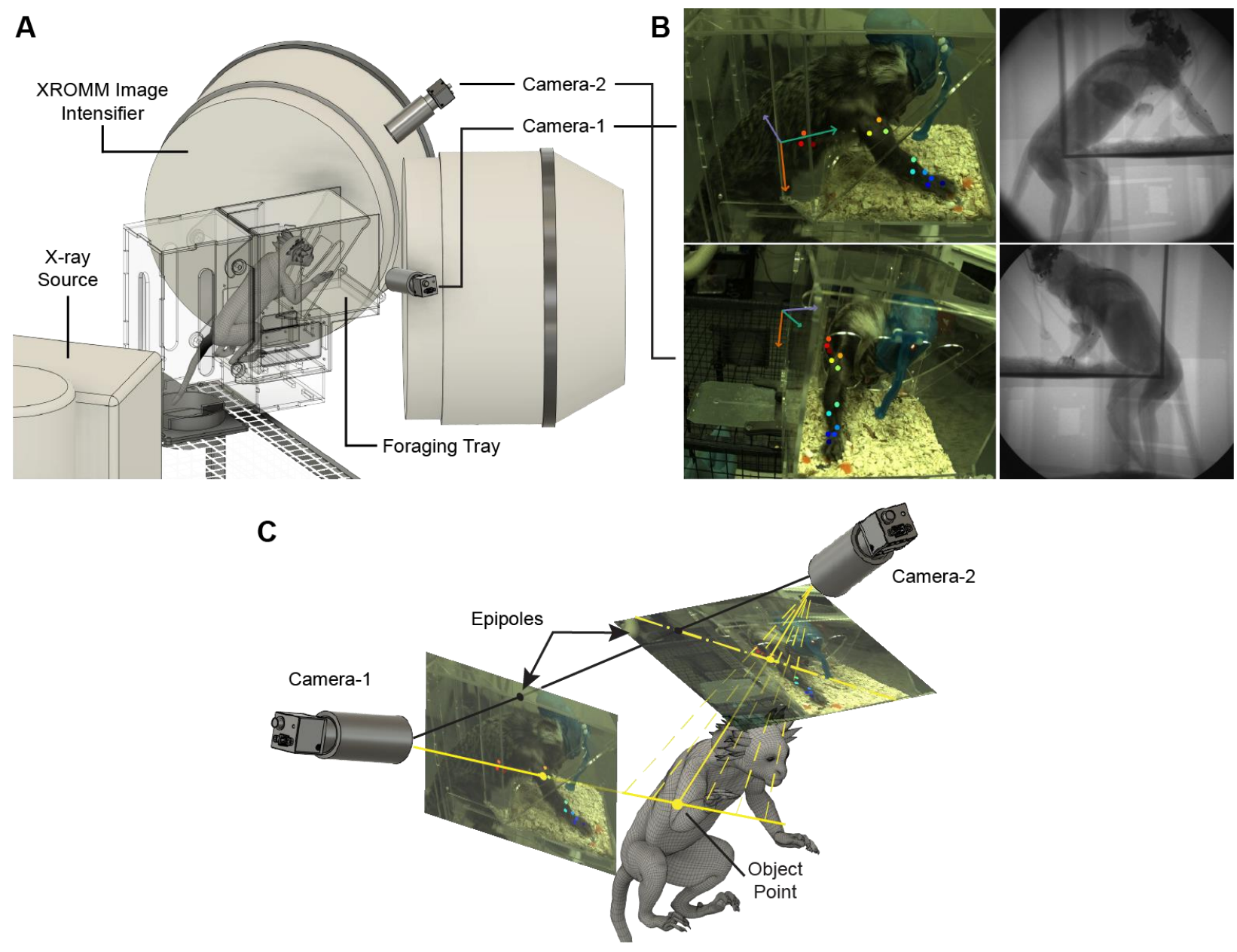

311 Figure 1: Recording apparatus, markers, and labeling with epipolar lines. A) Marmosets use their upper limb to forage. Blackfly $S$ cameras and XROMM record foraging behavior simultaneously. B) Left: Videos from camera-1 (top) and camera-2 (bottom). Markers are applied by DLC. The coordinate system generated by camera calibration is shown in green (x), orange (y), and purple (z). Right: corresponding frames from XROMM. Markers are tantalum beads implanted subcutaneously, seen as small black dots and tracked offline with XMALab. lines improve labeling accuracy. A vector projects from camera-1 through the applied label into 3D space, where it intersects with possible vectors from camera-2 (a subset is shown in dotted yellow). The epipolar line (dot-dash) passes through the epipole in camera-2 and each of the points at which a vector intersects the image plane. A correct label applied along the epipolar line produces accurate triangulation to the object point (correct labels and camera-2 vector shown in solid yellow). 
A
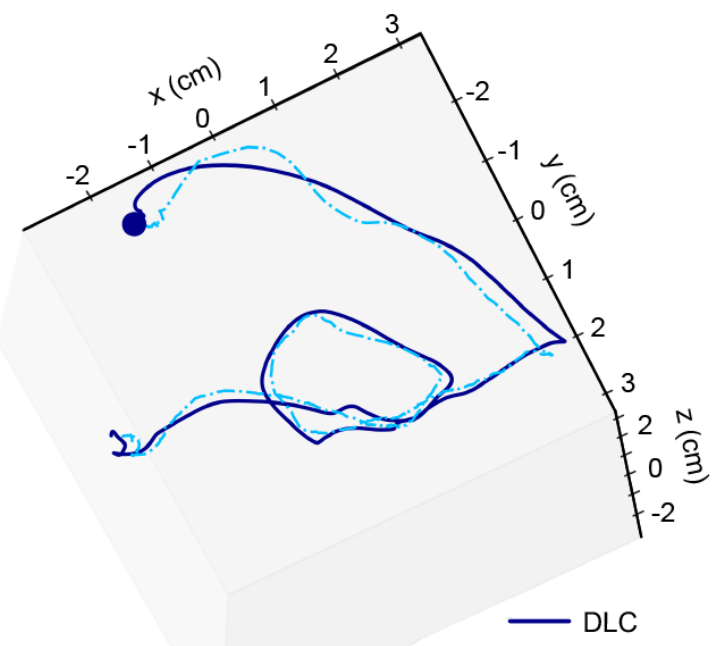

$-\cdot-$ XROMM
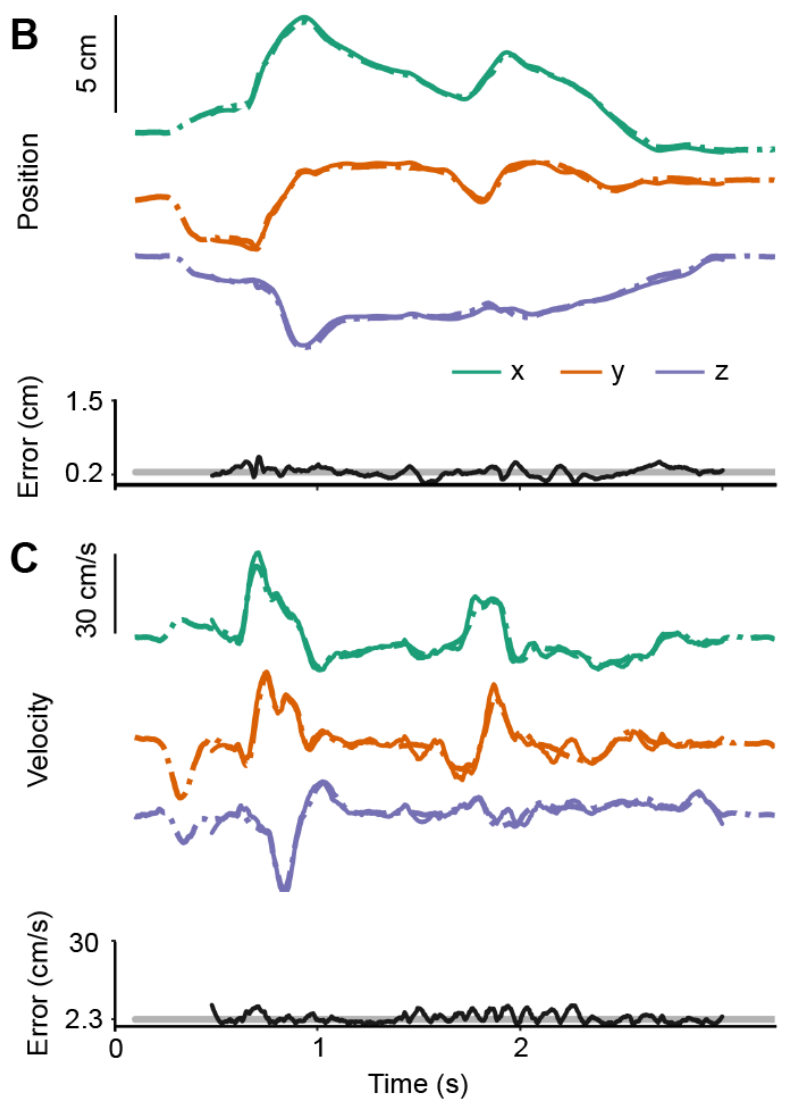

D

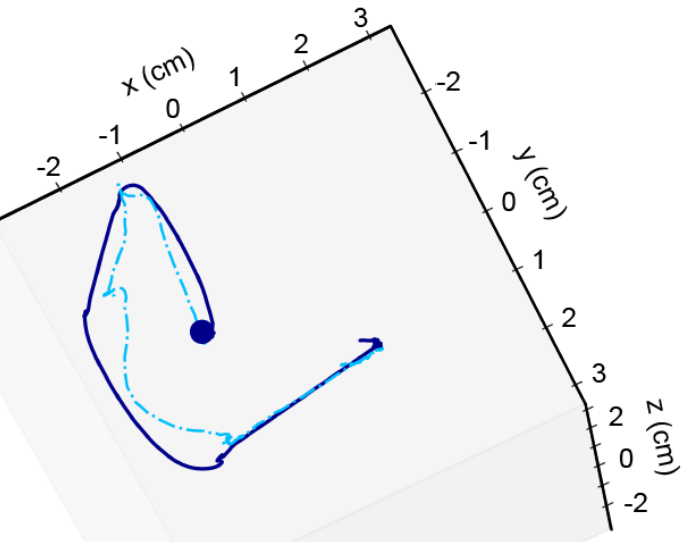

$\mathbf{E}$
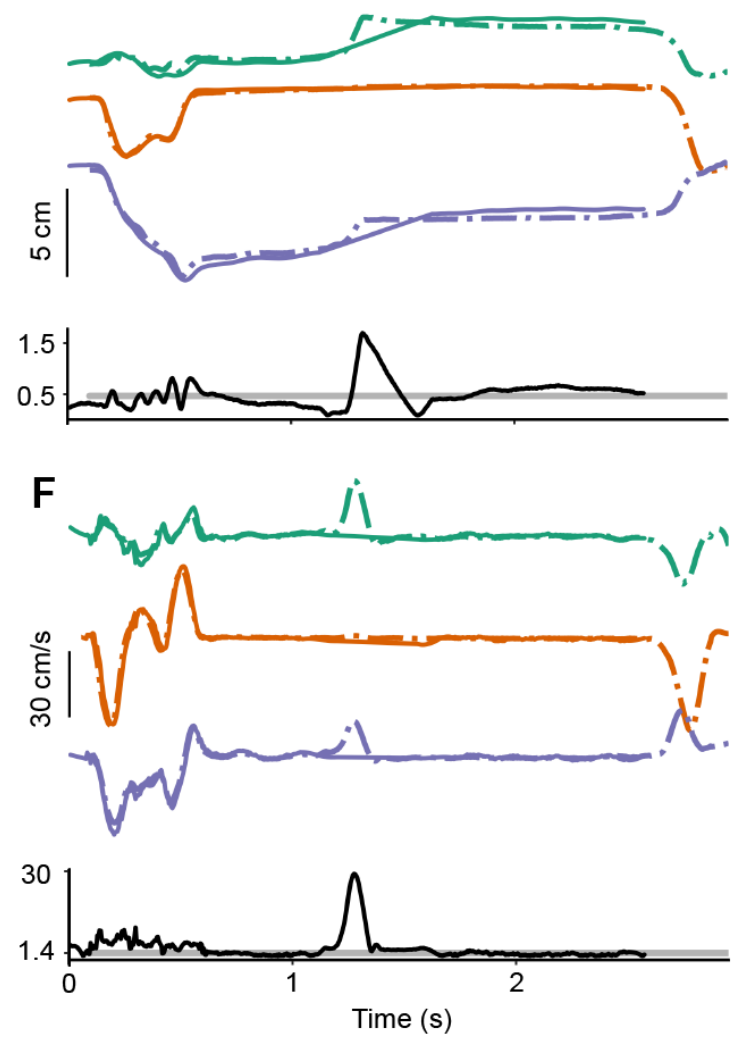

Figure 2: Tracking position and velocity with DLC and XROMM. A-C) Example of accurate DLC tracking. A) 3D position of distal hand marker with DLC (dark blue, solid) and XROMM (light blue, dot-dash). The plot is rotated to approximate the DLC camera-1 angle. B) Detailed position. Top: kinematics split into $x-y-z$ coordinates colored to match the axes in Fig. 1B, with DLC in solid and XROMM in dot-dash. XROMM extends before and after DLC because we filtered out DLC when the hand is well behind the foraging partition. Bottom: Error at each time 
bioRxiv preprint doi: https://doi.org/10.1101/2021.06.15.448541; this version posted June 16, 2021. The copyright holder for this preprint (which was not certified by peer review) is the author/funder. All rights reserved. No reuse allowed without permission.

330 (black) overlaid on median error for the event (gray). C) Velocity for same event. D - F)

331 Example of the errors produced by DLC. The marker movement missed by DLC at $\sim 1.25 \mathrm{~s}$ came

332 from removing a marker jump during a movement and replacing it with smoothed interpolation. 

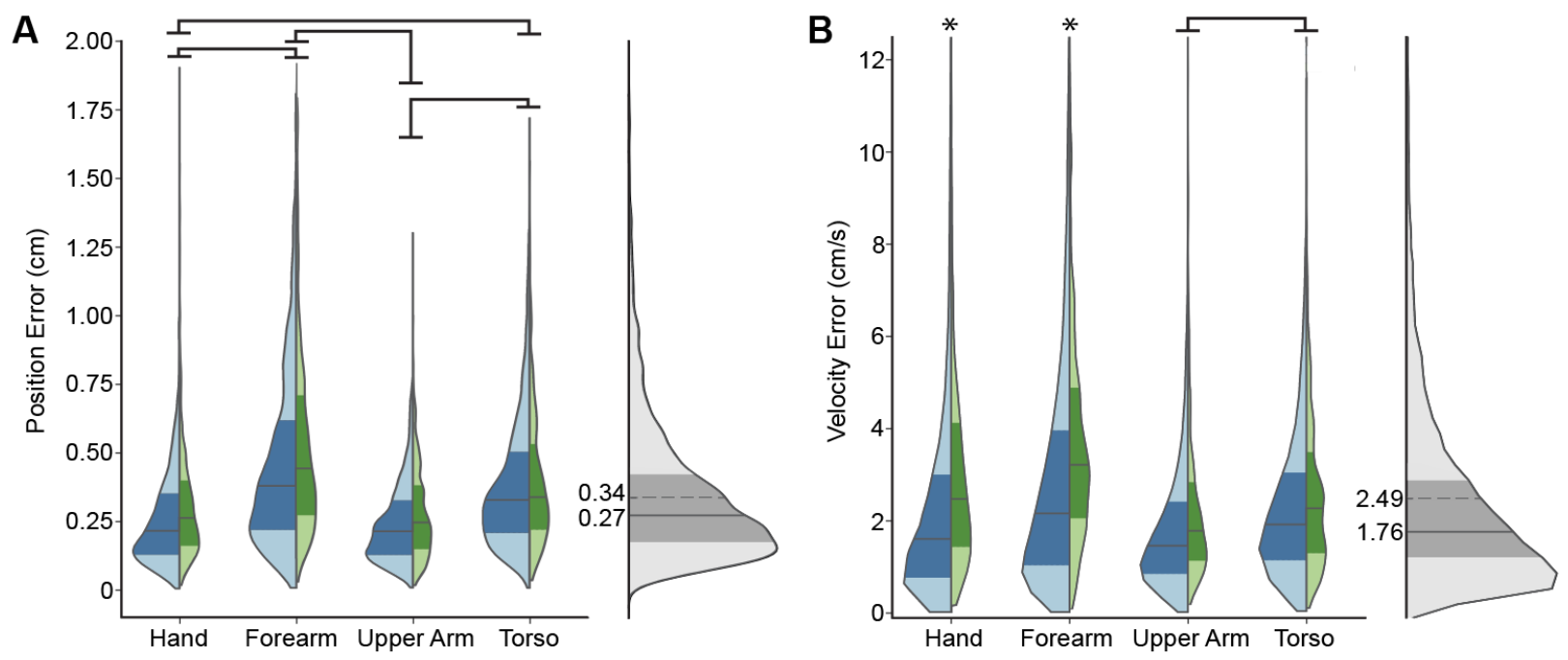

Figure 3: Error distributions. A) Position error distributions. Left: violin plots of absolute error separated by body segment. Each segment contains three DLC-XROMM marker pairs. Errors for 169 training frames are in green, with 5,721 test frames in blue. Median and interquartile range are shown by a gray line and shading. Significant effects with $r>0.20$ are indicated by bars for segments and asterisks for test-train. Right: distribution of pooled errors using the same conventions for median and interquartile range, and mean error at the dotted line. B) Velocity error distributions. 